\title{
Classification of quality conditions of a traction motor frame insulation of locomotives
}

\author{
Michail Kapitsa ${ }^{1}$, Vasiliy Laguta ${ }^{2, *}$, and Yuriy Kozik $^{3}$ \\ 1 Dnipro National University of Railway Transport named after Academician V. Lazaryan, Department of Locomotives, \\ 49010 Dnipro, Lazaryan Street 2, Ukraine \\ 2 Dnipro National University of Railway Transport named after Academician V. Lazaryan, Department of automation and \\ telecommunications, 49010 Dnipro, Lazaryan Street 2, Ukraine \\ ${ }^{3}$ LLC "Applied Railway Technologies", Head of Technical Projects, Department of Inspectors, Adam-Klein-Str. 26, Nürnberg D- \\ 90429, Germany
}

\begin{abstract}
The objective of the article is to determine the quality conditions of a locomotive traction motor frame insulation based on observations over the recovery voltage and classification of a tested insulation wear degree as well as to determine estimates of operating time until the insulation restoration or replacement with a new one. To classify the insulation quality conditions and to forecast its residual life until the restoration, a cluster analysis and discriminant analysis are used based on indicators reflecting the properties of an insulation recovery voltage curve. The study defines an information content received from frame insulation recovery voltage readings as compared with its resistance from the perspective of testing the insulation condition and predicting its residual life. Statistical characteristics of clusters corresponding to the insulation condition stages allow determining the limit values of predicting parameters and estimating the residual insulation life prior the restoration. To classify the insulation condition, an integrated evaluation of the insulation condition quality is proposed. Integrated evaluation is performed according to the recovery voltage curve and is used as an indicator during clustering. An experimental study of the proposed method practically confirmed its effectiveness.
\end{abstract}

\section{Introduction}

The analysis of "Ukrzaliznytsia" (Ukrainian Railways) locomotive fleet condition shows that the amount of damage happened to the TM winding insulation per one $\mathrm{mln} \mathrm{km}$ of run has recently averaged from three to five cases. Therefore, an increased attention is given to the problem of improving the operational reliability of TM insulation.

Systems of maintenance, repair and in-between diagnostics in the locomotive economy of Ukrainian railways are periodic and subject to regulation. To perform the majority of measurements the equipment should be withdrawn from the operation. However, such diagnostic system does not meet modern requirements. Continuous monitoring of the TM insulation technical condition becomes an optimal solution enabling reorganizing of the operational diagnostics. Automated continuous control along with predicting has several advantages over the periodic control, i.e. the reduction of checking periods, consideration of effects caused by operational factors, accumulation of diagnostic information, remote provision of information at various levels, etc. The introduction of automated monitoring will allow scheduling of equipment repairs and, if necessary, urgent TM withdrawing from operation. This approach will allow transferring from the outdated system of periodic maintenance to the maintenance matching the real technical condition of an object. Continuous monitoring systems need specific procedures, algorithms and models necessary for their effective functioning.

The condition of frame insulation of winding located at the main poles of traction electrical machines (hereinafter referred to as frame insulation) is a serious factor ensuring reliable TM operation. The implementation and development of works in respect of the frame insulation service life management was stimulated by the decision to extend the design service life of locomotives. Important elements of the service life management programme are non-destructive diagnostics of the insulation condition and prediction of its service life. Improving methods for predicting the insulation condition implies the clarification of allowed values of aging coefficients.

One of the methods that allows possible predicting of the frame insulation residual life may be the procedure of classification of its condition according to the values characterizing the recovery voltage curve. The article offers a procedure of classification, classification values, allowed values of parameters for predicting the residual life of TM NB-406 frame insulation.

Corresponding author: vasilij.laguta@gmail.com 


\section{Literature review}

Aging and degradation of the insulation placed in electrical machines determine operational service life of the latter. Aging processes depend on the nature and values of electrical stresses, mechanical forces applied, operating temperatures and environmental conditions affecting (permanently or temporarily) the insulation.

The dynamic assessment of insulation conditions is given and highlighted in. Authors indicate that during the insulation aging process such phenomena as aging, degradation and destruction can be observed. Aging and degradation of insulation directly affect the destruction of insulation, but there is no clear line between aging and degradation. Differences between the phenomena of degradation and breakdown (sudden failure) of insulation are also given in [1]: "The essential difference between the degradation and breakdown phenomena consists in the fact that the degradation is a process that takes place for a long period of time, while the failure is a process that occurs suddenly and it is catastrophic, insulation being unable to support the nominal voltage after its failure".

In $[2,3]$ changes in the insulation quality condition is associated with the process of aging and is considered from the molecular physics perspective. Molecular changes in the insulation contribute to the deterioration of electrical, mechanical and other properties thus leading to the development of mechanisms of insulation degradation.

The specific requirements in respect of the maintenance of locomotives running on the railways of Ukraine necessitate a slightly different approach to determining the stages of the electrical machines insulation quality in time than the approaches considered above. Regarding the frame insulation, to define its aging stages it is necessary to determine the type of restoration technological operation:

- to restore the frame insulation through soaking treatment;

- to replace the frame insulation with a new one.

The automatic diagnostics of the insulation of traction motors attracts more and more attention due to the accelerated insulation degradation observed in recent decades caused by the use of electronic pulse-width static converters in new types of rolling stock that generate powerful electromagnetic interference in a wide frequency range [4].

Several methods of dielectric diagnostics were developed to assess the insulation condition: polarization and depolarization current measurement (PDC polarization and depolarization current) [5], frequency domain spectroscopy (FDS) [6], recovery voltage measurement (RVM), [7, 8]. Measuring of the recovery voltage is a good method for obtaining information on the insulation condition.

Insulation residual resource can be determined via mathematical modeling of the insulation aging process.

Models $[9,10,11]$ are designed bearing in mind insulation structural changes occurred due to electromechanical stresses capable to destruct crystallites or damage interfaces in composite materials.

In model [12], authors proceed from the fact that a space charge formed in the insulation sooner or later becomes the cause of aging. The charge accumulated in different insulation zones locally increases the electric field, increases the mechanical stress, and then chemical bonds are destroyed.

The model presented in [13] proceeds from the assumption that a space charge is formed because of aging, and the aging process itself is due to high electric fields that destroy chemical bonds in the insulation.

The most favourable models for predicting the residual insulation resource still are statistical models.

Models that use neural network modelling of insulation condition diagnosing should be distinguished among the statistical models [14]. This work applies models based on feed-forward artificial neural networks. In [15] researchers propose exponential models and models based on Weibull's distribution law. To determine the residual resource the regression models are used as well. Cox's regression model [16] is the most common regression model since it is not related to assumptions on the tolerance time distribution. The model assumes that the resource wear process occurs independently of operational impacts.

The disadvantages of statistical models include the fact that all of them require a large amount of reliable statistical data.

\section{Materials and Methods}

\subsection{Analysis of VL-8 locomotive TM failures (depot Nizhnedneprovsk-junction)}

Damaged VL-8 locomotives were observed at depot Nizhnedneprovsk-junction railway yard (Ukraine). The prerequisite for selecting locomotives to be statistically relevant was the fact that locomotives underwent complete overhaul (CR) and TMs were fully restored. Locomotive traction motors are characterised with fairly high level of failure, i.e. $12 \%$ (provided that a locomotive has 6 elements). Almost a third of TM failures are failures of insulation of TM excitating windings, Fig. 1.

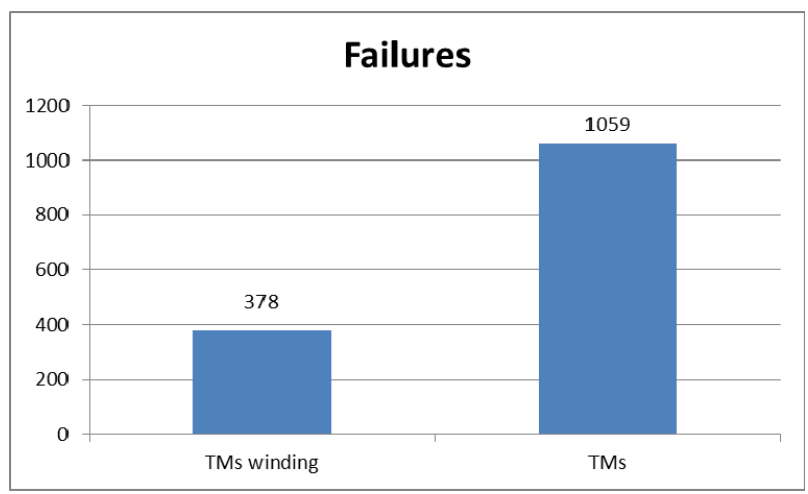

Fig. 1. Failures of winding insulation relative to the total TM failure rate. 
Fig. 2 graphically represents the dependence of failures of TM excitating winding isolation and the TM itself.

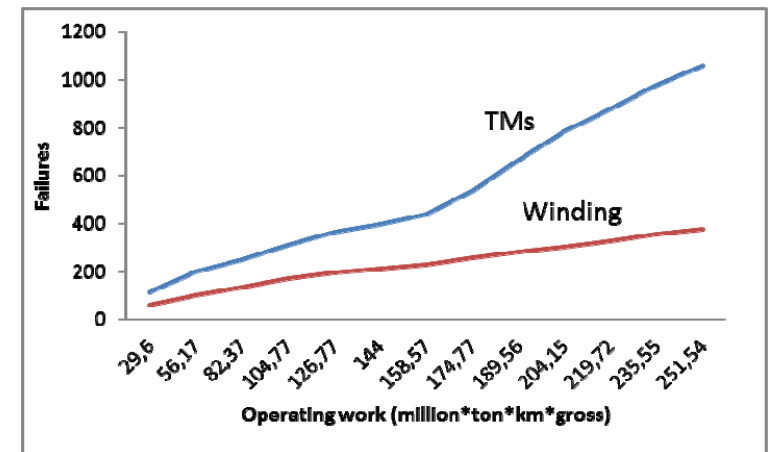

Fig. 2. Dependence of TM winding isolation failures and TM failures in relation to operating work (million*ton*km*gross).

Table 1 indicates dynamic failures of major structural elements of a TM. Special attention should be paid to the $\mathrm{TM}$, where a tendency of failure increase is observed.

Table 1. Percentage-wise ratio of unplanned repairs of traction motors per their major structural elements

\begin{tabular}{|l|c|c|c|c|}
\hline TM element & $\mathbf{2 0 1 4}$ & $\mathbf{2 0 1 5}$ & $\mathbf{2 0 1 6}$ & $\mathbf{2 0 1 7}$ \\
\hline Armature & 70,5 & 73,5 & 69,5 & 64,4 \\
\hline Pole winding & 11,6 & 8,55 & 10,5 & 16,8 \\
\hline Cable connections & 8,53 & 6,84 & 16,8 & 9,9 \\
\hline $\begin{array}{l}\text { Motor armature } \\
\text { bearings }\end{array}$ & 9,3 & 11,1 & 3,16 & 8,91 \\
\hline
\end{tabular}

Measurements were performed and research was carried out in respect of the insulation resistance change in TM main poles excitation winding depending on the run during summer and winter periods. It turned out, that the insulation resistance, measured by using a megohmmeter with a relative accuracy, does not demonstrate any established regularity and is not subject to any descriptive law. That is, this parameter is not informative in terms of diagnostics and prediction of the coil insulation condition. Several attempts were made to establish the dependence of insulation resistance on the run and seasonal "winter-summer" use (Fig. 3, 4), which also did not yield the expected results.

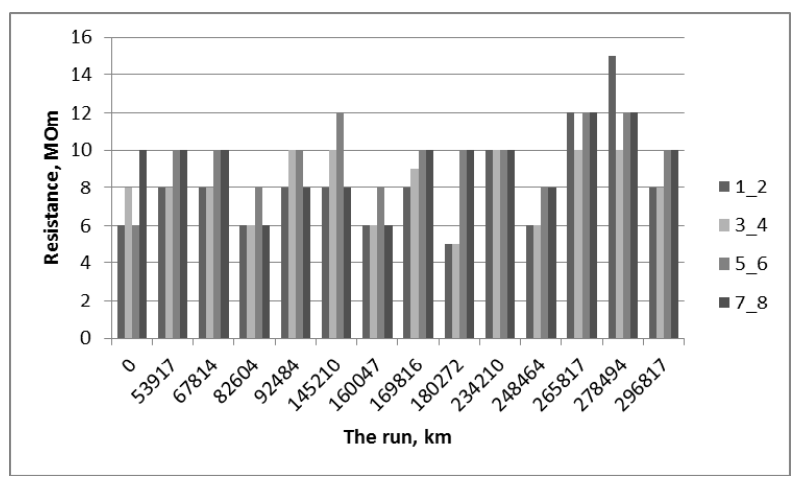

Fig. 3. Changes in TM main poles excitation winding insulation resistance depending on the run (summer). $12,3 \quad 4$, 5_6, 7_8-poles.
Measurement of insulation resistance by using a procedure recommended in "Rules for Locomotive Repairing" is to only control this parameter so that it does not exceed the allowed limits. To obtain an informative diagnostic parameter, which could be used to make conclusions on the insulation condition at a given time and to predict changes in its future condition, it is necessary to develop new procedures and methods.

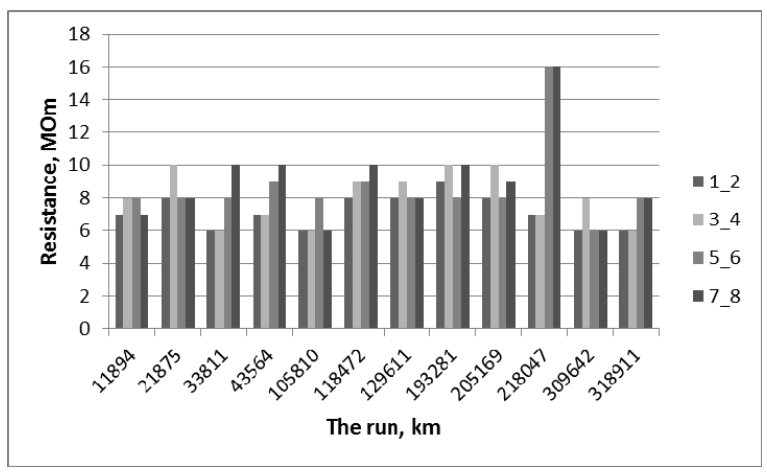

Fig. 4. Changes in TM main poles excitation winding insulation resistance depending on the run (winter). 1_2, 3_4, $5 \_6,7 \_8$ - poles.

Measures to eliminate TM winding failures will increase the reliability of $\mathrm{TM}$ and reduce penalties charged due to work failures, demanded by operational departments from the depot.

\subsection{Problem statement}

The vast majority of known insulation resistance measuring methods and devices are designed to be used in an auto-mode. In connection with this, methods for assessing the insulation condition are necessary, which allow for both autonomous and continuous monitoring of the insulation condition. Despite the considerable amount and variety of existing methods and devices, no classification of the insulation wear share is available, which makes it difficult to decide on the timing of its restoration.

The objective of this work is to determine quality conditions of the traction motor frame insulation according to the recovery return voltage data based on the classification of the wear level of the insulation being diagnosed, as well as to determine operating run estimates until the restoration or replacement of the insulation with a new one.

\subsection{Integrated evaluation of the insulation quality condition}

An integral evaluation can take into account not only the magnitude of some process, but also the rate of its change, etc. Integrated evaluation is intended to produce a general evaluation of the damping rate and a value of the process deviation from a given value without determining the former and the latter individually. The simplest integral evaluation can be an area between the values of the studied process and its steady state value. 
The main characteristic used to determine indicators of the insulation condition is the recovery voltage. The recovery voltage yields the fullest information on the insulation aging process.

Characteristics of the insulation condition are conditionally divided into two types: direct and indirect. Direct characteristics include those, which are directly derived from the recovery voltage plot (measurements). Indirect characteristics include those, which are calculated based on the recovery voltage observational data. An integral evaluation of the insulation condition is attributed to the indirect characteristic. We will select and define the characteristics based on the information on recovery voltage observational data.

Integrated evaluation of the insulation condition allows characterizing the recovery voltage curve with a certain number, which comprehensively evaluates the curve as a whole over a given interval. As a rule, such evaluations are functionals and are expressed as integrals.

Let us have a look at some integral characteristics. Since the conditions of measurements can be different at different times (voltage supply instability, meteorological conditions, etc.), the sequence of measured recovery voltage values should be normalised. The range of recovery voltage values $U_{k}, k=\overline{1, n}$ should be reduced to a single interval $u_{k} \in[0,1]$ using dependence

$$
u_{k}=\frac{U_{k}-U_{\min }}{U_{\max }-U_{\min }}, k=\overline{1, n},
$$

where $u_{k}$ is a normalised value of $k$-th measurement; $U_{k}$ - is measured value of recovery voltage; $U_{\min }$ and $U_{\max }-$ minimum and maximum values of return voltage respectively; $n$ - number of measurements. Considering the peculiarity that $U_{\text {min }}=0$, then equation (1) will be simplified down to

$$
u_{k}=\frac{U_{k}}{U_{\max }}, k=\overline{1, n}
$$

Normalised values $u_{k}$ should be approximated using dependence $u(t)$ in measurement interval $t \in\left[0, t_{C}\right]$. In the study, the approximation of measurement of the recovery voltage was performed using cubic splines.

Integral indicator of the insulation condition $J_{0}$ in based on the work evaluation, which is done by the insulation dipoles under the influence of a constant electric field. The magnitude characterizing the work of dipoles may be limited by a recovery voltage curve and the time axis. Formally, the recovery voltage drop time $U(t)$ goes to the infinity. The evaluation of work of dipoles at self-discharge, evaluation time interval $t_{C}$ should be chosen in such a way as to allow $U\left(t_{C}\right)=U_{\max } / 2$, where $U_{\max }-$ is the recovery voltage maximum value. Now, we can yield the target evaluation from the equation

$$
J_{0}=\int_{0}^{t_{C}} u(t) d t
$$

A similar way of evaluating di-electrical properties is considered when determining the reliability of capacitors.

Integrated evaluation (3) takes into account only the additive quality of the recovery voltage curve. An integral evaluation of the recovery voltage curve can be made via evaluating

$$
J_{01}=\int_{0}^{t_{C}}\left[u(t)+\gamma \cdot u^{\prime}(t)\right] d t, \gamma>0 .
$$

Integrated evaluation (4) takes into account both additive quality of the recovery voltage curve and the curve change rate (recovery voltage growth rate and the rate of self-discharge). It should be noted that normalising of a scale for the recovery voltage derivative $u^{\prime}(t)$ is performed in the same way as for the recovery voltage measurements.

The assessment of recovery voltage curve quality also can be performed using an integral quadratic criterion

$$
J_{21}=\int_{0}^{t_{C}}\left[u^{2}(t)+\gamma \cdot\left(u^{\prime}(t)\right)^{2}\right] d t, \gamma>0
$$

or

$$
J_{12}=\int_{0}^{t_{C}}\left[u(t)+\gamma \cdot\left(u^{\prime}(t)\right)^{2}\right] d t, \gamma>0,
$$

parameter $\gamma$ is a calibration parameter. It is chosen depending on the importance of a derivative (summand).

A type of equation used for the integral evaluation depends on the type of frame insulation and is chosen experimentally. In general, the insulation quality will be characterized by two indicators $\left(J, t_{C}\right)$, where $J$-is an integral evaluation of the recovery voltage curve; $t_{C}$-time of curve evaluation (the upper limit of integration of the integral evaluation equation). As an integral evaluation value we can take one of values $J_{0}$, $J_{01}, J_{21}, J_{12}$.

\subsection{Identification of classes of NB-406 TM frame insulation quality conditions}

When predicting using the method of classification, it is necessary to solve two problems: first, to build a set of classes; secondly, it is necessary to evaluate the attributes and, according to the results of evaluation, to relate an object of predicting to this or that class. Solving of the first task requires processing a large amount of statistical data obtained during the operating period and using technical 
objects or performing special experiments. The possibility of forming classes largely depends on the successful selection of diagnostic attributes. These attributes should sufficiently enough characterize the course of processes leading to the loss of the object's workability, and their accurate evaluation should not present great difficulties. The success of solving the second task is largely determined by the accuracy of relating an object to a known class based on the evaluation results. The class is characterised by a certain tendency of change occurred to the object to over time.

In the study the insulation quality is characterised by a pair of indicators $\left(J_{01}, t_{C}\right)$, where $J_{01}$ - integral evaluation of the recovery voltage curve calculated in accordance with equation (4), $t_{C}$ - time of recovery voltage curve evaluation. The distribution per clusters were performed using the method of balls. Isotonic and isomorphic distribution per clusters was used. Clustering was carried out for 38 curves and allowed defining three groups of curves (clusters), i.e. insulation conditions. Upon clustering 7 curves were removed from the received clusters.

The recovery voltage curves were constructed for the frame insulation showing different degrees of wear. In addition, the statistics included objects with the restored frame insulation of the main poles winding.

\section{Results and Discussion}

In accordance with the results obtained during the cluster breakdown procedure, we introduce the following stages of the frame insulation condition: 1st stage (cluster $K_{1}$ ) - "satisfactory condition"; 2nd stage (cluster $K_{2}$ ) "restored condition"; 3rd stage (cluster $K_{3}$ ) - "high risk condition". The introduced stages are conditional and may be interpreted as follows. The first stage means new insulation or insulation being used for a negligible period or its condition may be referred to as "new". As a rule, such insulation does not need substantial maintenance measures during technical check-up. The second stage means insulation, which is characterised by the wear of almost a half of operating run until the first failure. Regular restoration of the insulation is recommended at this stage. The third stage is characterised by an increased risk of insulation failure occurrence (ranging from the increased possibility of partial discharge to the insulation breakdown). At this stage, the last restoration or the complete restoration of the insulation should be planned (replacement of frame insulation). This work does not consider the moment of a complete wear. We presume that when the recovery voltage reaches $40-50 \mathrm{~V}$ at 25 th second of measurement, insulation should not be restored and is subject to be replaced with a new one. Further usage of such frame insulation can cause its failure while a locomotive is moving. In accordance to the introduced insulation condition stages, a set of recovery voltage curves, which can be referred to them, will be called as follows: the 1st curve group, the 2nd curve group, the 3rd curve group. Fig. 5 shows examples of recovery voltage curves: with maximum $U_{\max }$ voltage (the 1st curve group), with minimum $U_{\max }$ voltage (the 3rd curve group), a curve from the second curve group is in the middle of the Fig. 5.

Using other methods of clustering it is possible to obtain a different number of clusters (insulation conditions). The study used integral evaluations (5) and (6) as indicators that differ from the above (4), but in this case, the distribution of curves per clusters did not correspond to experimental observations.

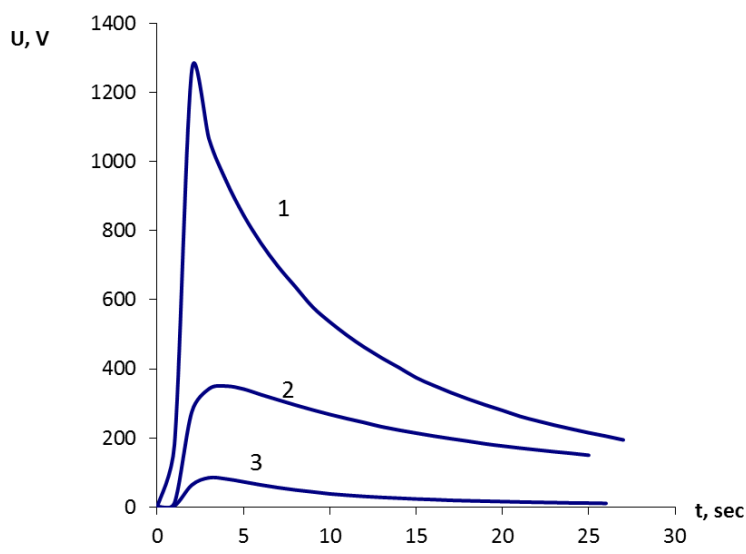

Fig. 5 - Example of the recovery voltage curves at various stages of insulation condition:

1 st curve corresponds to the 1 st stage, i.e. "satisfactory condition"; 2nd curve corresponds to the 2nd stage, i.e. "restored condition"; 3rd curve corresponds to the 3rd stage, i.e. "high risk condition".

Calculation of values of critical radii $R_{i}$ to determine a cluster which a new object belongs to (recovery voltage curve of the diagnosed frame insulation) was performed according to the method of balls.

For received clusters the values of classification indicators should be averaged, $\bar{J}_{i}=\overline{\tilde{J}}_{01}, \tilde{J}_{01} \in K_{i}$ and $\overline{t_{i}}=\overline{\tilde{t}}_{C}, \tilde{t}_{C} \in K_{i}$. Values $\tilde{J}_{01}$ and $\tilde{t}_{C}$ are respective normalized values, similar (1)-(2), integral evaluation $J_{01}$ and time $t_{C}$. Operating run $\bar{T}_{i}$ of frame insulation coils is averaged as well since it falls into the studied cluster of curves $K_{i}, i=\overline{1,3}$.

Verification of the normal law of distribution of values of operating run of frame insulation for the constructed clusters was carried out.

The verification of the distribution law has shown that operating run values demonstrate normal distribution characteristics with the level of significance $\alpha=0,05$. For operating run averaged values $\bar{T}_{i}$ confidence intervals $\left(\bar{T}_{i}-\Delta T_{i}, \bar{T}_{i}+\Delta T_{i}\right), i=\overline{1,3}$ were defined. The value $\bar{T}_{i}$ can be interpreted as an average operating run at which the frame insulation reaches $i-$ th condition. Cluster parameters and confidence intervals of insulation operating run for each cluster are presented in Table 2. 
Table 2. Characteristics of classification clusters used to determine isolation quality conditions

\begin{tabular}{|l|c|c|c|c|c|}
\hline \multicolumn{1}{|c|}{ Condition } & $\bar{J}_{i}=\overline{\tilde{J}}_{01}$ & $\overline{t_{i}}=\overline{\tilde{t}}$ & $R_{i}$ & $\begin{array}{c}\left(\bar{T}_{i}-\Delta T_{i}, \bar{T}_{i}+\Delta T_{i}\right) \\
\text { thnds km }\end{array}$ & $\begin{array}{c}\text { Point of restoration } \\
\text { according to the } \\
\text { repair rules, thnds km }\end{array}$ \\
\hline 1st stage & 0.87 & 0.43 & 0.15 & $(165,405)$ & 350 \\
\hline 2nd stage & 0.71 & 0.74 & 0.26 & $(580,940)$ & 700 \\
\hline 3rd stage & 0.13 & 0.54 & 0.17 & $(865,1175)$ & 1050 \\
\hline
\end{tabular}

The quality condition of the diagnosed insulation (new diagnosed object) and values of the recovery voltage curve $\left(\tilde{J}, \tilde{t}_{C}\right)$ are calculated if the following precondition is met

$$
\left(\tilde{J}-\bar{J}_{i}\right)^{2}+\left(\tilde{t}_{C}-\bar{t}_{i}\right)^{2}<R_{i}^{2}, i=\overline{1,3}
$$

If the fulfilment of inequality (7) is not unique, then the object's membership in a cluster is defined using a smaller evaluation

$$
\Delta_{i}=\left|\left(\tilde{J}-\bar{J}_{i}\right)^{2}+\left(\tilde{t}_{C}-\bar{t}_{i}\right)^{2}-R_{i}^{2}\right|, i=\overline{1,3} .
$$

The normalisation of indicators $\left(J, t_{C}\right)$ of a new object being tested is performed within the whole set of indicators $\left\{J_{i}\right\}_{i=\overline{1, N}}$ and $\left\{t_{C i}\right\}_{i=\overline{1, N}}$ respectively, $N$ - the number of indicators - the sum of elements $\left(J, t_{C}\right)$ included in clusters $K_{i}, i=\overline{1,3}$. Normalisation (1)-(2) is fulfilled if only two inequalities are simultaneously true

$$
J \leq J_{\max } \text { and } t_{C} \leq t_{C \max } .
$$

Upon testing a new object it is necessary to add new object $\left(J, t_{C}\right)$ to the cluster, which the tested object belongs to and to re-calculate necessary statistical characteristics of the modified cluster.

If one of inequalities (9) is untrue, it is necessary to perform the classification once again taking into the new case isolation tested object with subsequent determining its resource.

Depending on the membership of a diagnosed object in some cluster, the insulation condition will be characterised by the operating run value until its current restoration (including the replacement of an old insulation for a new one). The operating run value until its first restoration $\tau_{1}$ is defined using equation

$$
\tau_{1}=\bar{T}_{1}+\Delta T_{1}-T, T<\bar{T}_{1}+\Delta T_{1},
$$

where $T$ - is current operating run workout of the diagnosed insulation;

evaluation of the operating run value until the second restoration $\tau_{2}$

$$
\tau_{2}=\bar{T}_{2}+\Delta T_{2}-T, \bar{T}_{1}+\Delta T_{1}<T<\bar{T}_{2}+\Delta T_{2}
$$

evaluation of the operating run value until the replacement of insulation for a new one or it complete renovation $\tau_{3}$

$$
\tau_{3}=\bar{T}_{3}+\Delta T_{3}-T, \bar{T}_{2}+\Delta T_{2}<T<\bar{T}_{3}+\Delta T_{3} .
$$

If inequality (7) is untrue for any cluster, then it is possible to evaluate the insulation quality condition by using its workout until the time of its restoration according to the repair rules defined with one of the following values

$$
\begin{gathered}
\tau_{1}=350-T, T<350 \\
\tau_{2}=700-T, 350<T<700 \\
\tau_{3}=1050-T, 700<T .
\end{gathered}
$$

To determine whether the studied recovery voltage curve belongs to this or that cluster is also possible via discriminant analysis. In this case, it is necessary to have available learning samples of insulation quality conditions (clusters are filled with test objects during the maintenance process).

The recognition whether the tested frame insulation belongs to a cluster in accordance with equation (7) - (8) should be applied at the initial stage. When clusters contain a sufficient number of elements, then it is more appropriate to perform discriminant analysis.

The above method of determining the quality condition of frame insulation is acceptable within one depot, where locomotives are usually operated in the same way.

\section{Conclusion}

The classification of recovery voltage curves allows determining frame insulation quality conditions.

Clustering attributes are as follows: $J$ - integral evaluation of a recovery voltage curve determined from equation (4), $t_{C}$ - time of recovery voltage curve evaluation.

The breakdown of recovery voltage curves of the frame insulation into clusters allowed distinguishing three stages of the insulation quality condition and mark them "satisfactory condition", "restored condition", 
"high condition".

Software for classification of insulation quality conditions and discriminant analysis for determining the insulation condition has been developed.

The research presented in this article made to order and funded by Pridniprovska Railway Company, Ukraine. The authors are grateful to the management of the Depo Nizhnedniprovsk-Uzel engineering department for their assistance in obtaining the necessary data for research.The authors also express their gratitude to V.M. Zubenko (the engineer of the testing center of Pridniprovska Railway Company) for test assistance of the TMs frame insulation. Authors would also like to thank O. Voznyak - PhD, associate professor, Lviv branch of Dnipro National University of Railway Transport, Ukraine, for informational support.

\section{References}

1. Notingher, PV \& Plopeanu, M, "Accelerated Development of Electrical Trees. Part I: Initiation of Trees", EEA - Electrical Engineering, Electronics, Automatics, Vol. 57, No. 4, pp. 11-19, (2009).

2. Crine, J-P \& Vijh, AK, "Molecular Approach to the Physico-Chemical Factors in the Electric Breakdown of Polymers", Applied Physics Communications, Vol 5, No. 3, pp. 139-63, (1985).

3. Lewis, TJ, "Ageing - A Perspective", IEEE Electrical Insulation Magazine, Vol. 17, No. 4, pp. 6-16, (2001).

4. V. I. Havryliuk, "Modelling of the return traction current harmonics distribution in rails for AC electric railway system", 2018 International Symposium on Electromagnetic Compatibility (EMC EUROPE), IEEE, pp. 251-254, (2018).

5. Junhao Li, Yanming Li, "Influencing Factors Investigation of Polarization and Depolarization Current Measurements on Assessment Aging Condition of Power Transformer", International Review of Electrical Engineering (IREE), Vol. 5 No. 6, pp. 2637-2643, (December 2010).

6. A. A. Shayegani, E. Gockenbach, H. Borsi, H. Mohseni, "Investigation on the Transformation of Time Domain Spectroscopy data to frequency domain data for impregnated pressboard to reduce measurement time", Electrical Engineering, Vol. 89 No. 1, pp. 11-20, (June 2005).

7. T. K. Saha, M. Darveniza, D. J. T. Hill, T. T. Ie, "Electrical and chemical diagnostics of transformer insulation part A: Aged transformer samples", IEEE Transactions on Power Delivery, Vol. 12, pp. 15471554, (October 1997).

8. Michail Kapitsa, Vasiliy Laguta, Yuriy Kozik, "Selecting the Parameters of The Diagnosis of Frame Insulation Condition in Electrical Machines of Locomotives", International Journal of Engineering \& Technology, Vol. 7 (4.3), pp. 110-114. DOI: 10.14419/ijet.v7i4.3.19718, (2018).

9. Jones, JP, Llewellyn, JP \& Lewis, TJ, "The contribution of field-induced morphological change to the electrical aging and breakdown of polyethylene", IEEE Transactions on Dielectrics and Electrical Insulation, Vol. 12, No. 5, pp. 951- 966, (2005).

10. Lewis, TJ, "Polyethylene under Electrical Stress", IEEE Transactions on Dielectrics and Electrical Insulation, Vol. 9, No. 5, pp. 717-29, (2002).

11. Rowe, SW, "Electrical Ageing of Composites: An Industrial Perspective", Proceedings of International Conference on Solid Dielectrics, Winchester, UK, July 8-13, pp. 401-406, (2007).

12. Dissado, LA, Mazzanti, G \& Montanari, GC, "Elemental strain and trapped space charge in thermoelectrical aging of insulating materials. Life modelling", IEEE Transactions on Dielectrics and Electrical Insulation, Vol. 8, No. 6, p 966- 971, (2001).

13. Mazzanti, G \& Montanari, GC, "Electrical aging and life models: the role of space charge", IEEE Transactions on Dielectrics and Electrical Insulation, Vol. 12, No. 5, pp. 876-890, (2005).

14. LS. Skrupskaya, A.O. Oliinyk, A.A. Sakhno, "Model building for current transformer paper-oil insulation state diagnostics", ISSN 2074-272X. Elektrotexnika $i$ elektromexanika, No. 2, pp. 48-51, (2014).

15. O.A. Sakhno, "The continuous monitoring and remaining life prediction system for high-voltage current transformers". - Manuscript. The thesis for Ph.D. degree obtaining in the specialty 05.14.02 Electric Power Stations, Grids and Systems. National University "Lviv Polytechnics", Lviv, pp. 7-14, (2012).

16. Cox D.R., "RegressionModels and Life-Tables M", Journal of the Royal Statistical Society, Series B (Methodological), Vol. 34, No. 2, pp. 187-220, (1972). 